Research Article

\title{
Application of Molecular Dynamics in Coating Ag- Conjugated Nanoparticles with Potential Therapeutic Applications
}

Farzin Sohraby ${ }^{1}$, Mojtaba Hadi Soltanabad ${ }^{1}$, Milad Bagheri ${ }^{1}$, Masood Bezi Javan ${ }^{2}$, Mostafa Javaheri Moghadam $^{1}$, Eisa Kohan Baghkheirati ${ }^{3}$, Mohammad Bagher Bagherieh Najjar ${ }^{1}$

${ }^{1}$ Department of Biology, Faculty of Science, Golestan University, Gorgan, Iran.

${ }^{2}$ Department of Physics, Faculty of Sciences, Golestan University, Gorgan, Iran.

${ }^{3}$ Department of Biology, Hakim Sabzevari University, Sabzevar, Iran.

Corresponding author. E-mail: mb.bagherieh@gu.ac.ir Tel.: +98-17-32254161 Fax : +98-17-32245964

Received: Sep. 17, 2019; Accepted: Feb. 29, 2020; Published: Mar. 9, 2020.

Citation: Farzin Sohraby, Mojtaba Hadi Soltanabad, Milad Bagheri, Masood Bezi Javan, Mostafa Javaheri Moghadam, Eisa Kohan Baghkheirati, and Mohammad Bagher Bagherieh Najjar, Application of Molecular Dynamics in Coating Ag-Conjugated Nanoparticles with Potential Therapeutic Applications. Nano Biomed. Eng., 2020, I2(I): 90-98.

DOI: $10.5101 /$ nbe.v12i1.p90-98.

\begin{abstract}
Drug delivery systems may benefit from nanoparticles synthesized using biological methods. While chemical reduction of particles is facilitated by some active compounds present in the bio-extract, other active compounds, with potential therapeutic activities, may be adsorbed onto the surface of nanoparticles. However, the mechanism of bio-based nanoparticle synthesis is still under debate. Here, we first employed a molecular dynamics (MD) approach to theoretically predict the coating of a hypothetical $4.5 \mathrm{~nm}$ silver nanoparticle with four selected rosemary (Rosmarinus Officinalis L.) active compounds (rosmanol, isorosmanol, carnosol, and carnosic acid). Analysis of density maps and radial distribution functions (RDF) values suggested that the examined compounds had strong hydrophobic properties and could instantaneously be adsorbed to the nanoparticle surfaces. Next, we experimentally examined the capacity of rosemary leaf extract to synthesize and coat Ag-conjugated nanoparticles. The data obtained from ultraviolet-visible spectroscopy, transmission electron microscopy, Fourier-transform infrared spectroscopy and X-ray powder diffraction analyses confirmed the production of spherical Ag-conjugated nanoparticles with an average size of 12-15 nm, coated with proteins, secondary metabolites and other active compounds. Since this method can predict the dynamic behavior of therapeutic compounds when they are in contact with nanoparticles, we believe it provides a valid and new avenue to designing new therapeutic nanoparticles.
\end{abstract}

Keywords: Silver nanoparticle; Rosemary; Rosmarinus officinalis; Molecular Modelling; Molecular dynamics simulation

\section{Introduction}

Drug delivery has long been a concern in pharmacology. Nanoparticles, with various shapes and sizes, have been proved suitable candidates for delivering hydrophobic drugs and biologics through biological barriers [1-3]. Synthesis of nanoparticles
(NPs) using biological methods is considered to be safer, nontoxic, eco-friendly, and more affordable, as compared to physical or chemical methods [4-7]. Generally, in bio-based methods, natural compounds or extracts obtained from living organisms including bacteria, fungi and plants, containing chemical reducing activities, are employed in the synthesis 
of various NPs with different properties [8,9]. Interestingly, during this biosynthesis reaction, several natural compounds present in the bio-extract can be readily attached onto the surface of those synthesized NPs. Subsequently, by changing solvent properties, such as $\mathrm{pH}$, those compounds could be released from the surface of the NP. This phenomenon provides grounds for some interesting applications, including new means for isolation and purification of rare natural products, as well as new venues for drug delivery, using metallic NPs of choice, coated with selective therapeutic compounds.

Several plant secondary metabolites, including phenolic compounds and alkaloids are thought to be able to act as an electron donor to metallic ions, converting them to neutral NPs [10-12]. Rosemary (Rosmarinus officinalis) is a well-known evergreen member of the Lamiaceae family, which contains several secondary metabolites with strong antiplatelet [13], antioxidant [14, 15], anti-inflammatory $[16,17]$, anti-diabetic [18], anticarcinogenic [16, 19], antibacterial, and antifungal activities [20,21]. Among the various secondary metabolites present in rosemary leaf extract [22], carnosic acid, carnosol, rosmanol and isorosmanol are important diterpenes with potential valuable therapeutic applications [23, 24]; however, their properties and behavior in regard to attachment onto the surface of NPs have not yet been investigated.

Molecular dynamics (MD) simulation is a widely used tool in computational biology, with great capabilities in revealing the underlying mechanisms of biological processes such as chemical and dynamic properties of biological macromolecules as well as their interactions with each other and their surroundings [25]. Here, for the first time, a molecular dynamics (MD) approach has been exploited to analyze and model the molecular behavior of four hydrophobic compounds present in rosemary leaf extract, in Ag-conjugated NP biosynthesis. Subsequently, the proposed model was evaluated by experimental biosynthesis and characterization of NPs coated with rosemary active compounds.

\section{Experimental}

\section{Molecular dynamics simulation}

In order to perform the molecular dynamics simulations, a $4.5 \mathrm{~nm}$ quasi-spherical silver NP, containing $3781 \mathrm{Ag}$ atoms, kindly provided by Kyrychenko et al. [26], was placed in the center of a cubic box with a minimum distance of $1.5 \mathrm{~nm}$ from each side.

Using this box, in total, five various systems were prepared and simulated, in four of which, 20 molecules of each active compound were inserted individually. In the fifth system, four active compounds were inserted altogether at a quarter (5 molecules each) of the concentration used in their individual systems. The molecules were inserted into the box at random positions with a concentration of approximately 57 $\mathrm{mM}$.

All MD simulations were performed by GROMACS 5.1.2 software [27] using gromos53a6 force field [28] and SPC water mode [29], under periodic boundary conditions ( $\mathrm{PBCs}$ ). The whole complex was solvated by TIP3P water molecules. The systems were energy-minimized, using the steepest descent minimization algorithm for all atoms [30]. Each system was equilibrated in both NVT ensemble (constant number of particles $(\mathrm{N})$, volume $(\mathrm{V})$, and temperature $(\mathrm{T})$ ) coupled to the V-rescale thermal bath at $300 \mathrm{~K}$ over $100 \mathrm{ps}$ and in the NPT ensemble (constant number of particles $(\mathrm{N})$, pressure $(\mathrm{P})$, and temperature (T)) coupled to the Berendsen pressure bath at $1 \mathrm{~atm}$ over $300 \mathrm{ps}$. Each system was then subjected to a $30 \mathrm{~ns}$ molecular dynamics (MD) simulation under constant conditions of $1 \mathrm{~atm}$ and $300 \mathrm{~K}$ with a time step of $2 \mathrm{fs}$.

Bond lengths constrained using the LINCS algorithm [31] and the long-range electrostatics were applied using the particle mesh Ewald (PME) [32], while the SETTLE algorithm [33] was employed to constrain the geometry of water molecules. The trajectory information was analyzed using GROMACS utilities and VMD programs [34]. Moreover, UCSF chimera was used for visualization, inspection and preparation of the NP and compounds [35]. The force field parameters of the compounds were obtained from the ATB web server for gromos53a6 force field and the Lennard Jones parameters and the partial charge of Ag atoms were obtained from already published data $[26$, $36,37]$.

Although the actual partial charge of individual atoms in an Ag-conjugated NP molecule can dynamically vary between -0.07 and +0.07 [38], the net charge of the entire molecule is zero. To minimize the computing power needed for quantum mechanics calculations to an affordable value, the net- and partialcharges of the NPs were set to zero. 


\section{Biosynthesis and characterization of silver nanoparticles}

The leaves of Rosmarinus Officinalis L. were collected from Golestan University campus, Gorgan, Iran $\left(54^{\circ} 25^{\prime} \mathrm{E} ; 36^{\circ} 50^{\prime} \mathrm{N}\right)$ during 2012-2013. Samples were washed and air dried at $60{ }^{\circ} \mathrm{C}$ for $48 \mathrm{~h}$ and ground to a fine powder using an ordinary coffee grinder. One gram of each sample was used for extraction in $50 \mathrm{~mL}$ of ethanol for $48 \mathrm{~h}$ at room temperature. Afterwards, solutions were filtered through Whatman No. 3 paper and concentrated by a rotary evaporator at $<40{ }^{\circ} \mathrm{C}$. Silver-conjugated NPs were synthesized, as basically described by Hadi et al. [39]. 1\% (V/V) of the plant extract and $1 \mathrm{mM}$ of AgNO3 (SigmaAldrich, Germany) were mixed and retained at room temperature for $0,45,90,150,210$ or $300 \mathrm{~min}$. The solutions were then centrifuged at $20,000 \mathrm{~g}$ for $15 \mathrm{~min}$ and the resulting pellet was washed twice with distilled water and were left at room temperature for $16 \mathrm{~h}$ to dry before further characterizations. The progress of NP biosynthesis was monitored by measuring changes in the solution color, using an ultraviolet-visible spectrophotometer (Shimadzu UV-1800, japan) in the range of 200 to $800 \mathrm{~nm}$.

The crystallite structure of the particles was determined through recording their elemental spectra by an X-ray diffractometer (D8-Advance, Bruker, Germany) equipped with a $\mathrm{CuK} \alpha$ radiation source $(\lambda$ $=1.54 \AA$ ) in the range of $20^{\circ}$ to $80^{\circ}$ at a 0.5 degree $/ \mathrm{s}$ scan rate. The average crystallite size of the NPs was estimated by Sherrer's formula $(\mathrm{D}=0.9 \lambda / \beta \operatorname{Cos} \theta)$, where $\lambda$ stands for the $\mathrm{X}$-ray wavelength, $\beta$ for the full width at half-maximum and $\theta$ for the diffraction angle. The data were analyzed with XPowder software package. A Philips CM120 transmission electron microscope was employed to determine the size and shape of the synthesized NPs, as previously explained [39]. FTIR (Fourier-transform infrared spectroscopy) was performed using a Spectrum RXI (PerkinElmer, USA) in the range of $4000-400 \mathrm{~cm}^{-1}$ at a resolution of $4 \mathrm{~cm}^{-1}$, as described previously (40). The shape and size of the synthesized NPs were examined by a Philips CM120 transmission electron microscope at $120 \mathrm{kV}$ with a 2.5 Angstrom resolution, as described by the supplier, as described previously [11].

\section{Results and Discussion Molecular dynamics simulation}

The stability of the Ag-conjugated NPs was measured using the radius of gyration [41, 42], and is shown in Fig. 1. The radius of Ag NPs was slightly fluctuating while the $\mathrm{Ag}$ atoms vibrated in their positions during simulations. The stability of all NPs was declared by this method.

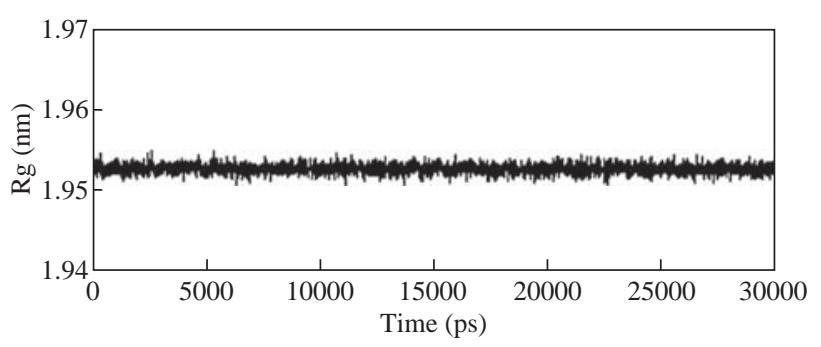

Fig. 1 The radius of gyration of Ag nanoparticle in one of the simulation systems.

The atomic motion and behavior of the compounds and their interactions with each other were studied by watching simulations' trajectories [43-46]. Our analyses showed that each of the active compounds in question was instantaneously attached to the NP (Fig. 2 and Supplementary movie 1). As shown in Fig. 2, all twenty molecules of each compound present in the box could be attached to the surface of the NP, either directly or indirectly, suggesting that increasing the concentration of the molecules present in the box resulted in the attachment of all compounds onto the NP until the entire surface was occupied.

The above-mentioned rosemary's four active

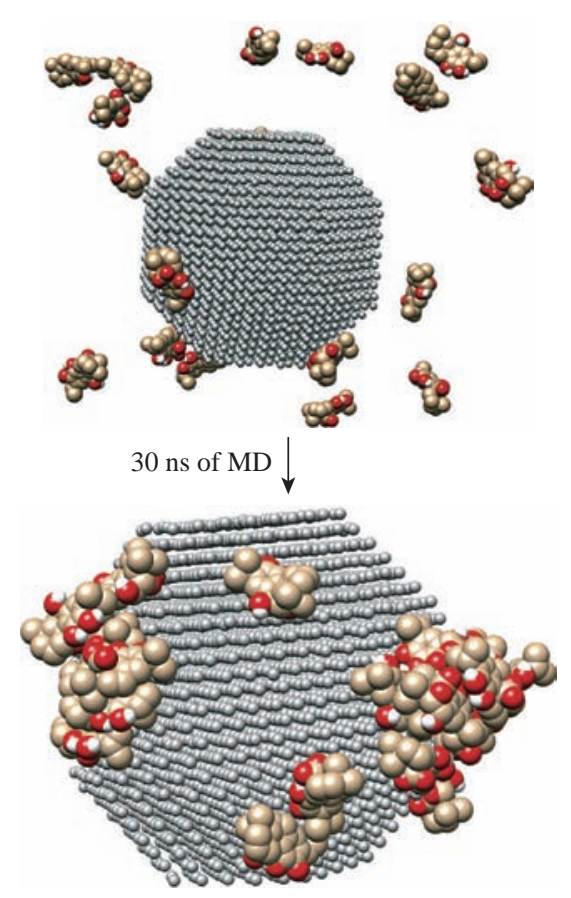

Fig. 2 The surface view of the Ag-conjugated nanoparticle after $30 \mathrm{~ns}$ of MD simulation. All of the 20 molecules could be absorbed on the surface of the nanoparticle. 
compounds (isorosmanol, carnosol rosmanol and carnosic Acid) have hydrophobic properties; i.e. they try to escape from interaction with the polar water molecules. This may explain why they immediately attached onto the NP surface or other compounds present in the solution (Supplementary movies 2 and 3). These four compounds were very similar in structure, and while all had functional hydroxyl and carbonyl groups, the three hydrophobic rings present in their structures dominantly made the compound's molecular behavior similar to a hydrophobic molecule, which resulted in the immediate interactions of rosemary's active compounds with each other and NPs.

In order to analyze the movements of active compounds relative to the target NP, the density map of ligands during the simulation process was calculated by utilizing the Gromacs densmap program. Gromacs densmap produced a 2D density plot of a selected group across the box (Fig. 3), which shows the presence-time of a chosen group in a specific location using color intensity (i.e. darker regions are indicative of longer presence). For clarity, we manually and schematically placed the Ag-conjugated NP on the density map. All four active compounds were attracted to the Ag NP, and maintained their positions during the simulation period (Fig. 3).

In addition, radial distribution functions (RDFs) were used to analyze the tendency of the active compounds to attach onto the Ag-conjugated NP surface. As shown in Fig. 4, the active compounds had the highest distribution density at $1 \mathrm{~nm}$ distance from the NP, which confirmed the attachment of all present onto the surface of Ag NP [47, 48].

In order to learn about possible key interactions influencing the attraction of active compounds to the NP surface, five separate MD simulations were performed. The results of these experiments showed that when the active compounds were approaching the NP, and their orientations were only affected by their initial orientation, while most of the molecules interacted with their hydrophobic rings [49] (Supplementary movies 4 and 5).

In MD simulations, assigning the accurate atomic partial charge of each atom is a key step to correctly modelling a molecular system behavior. Several
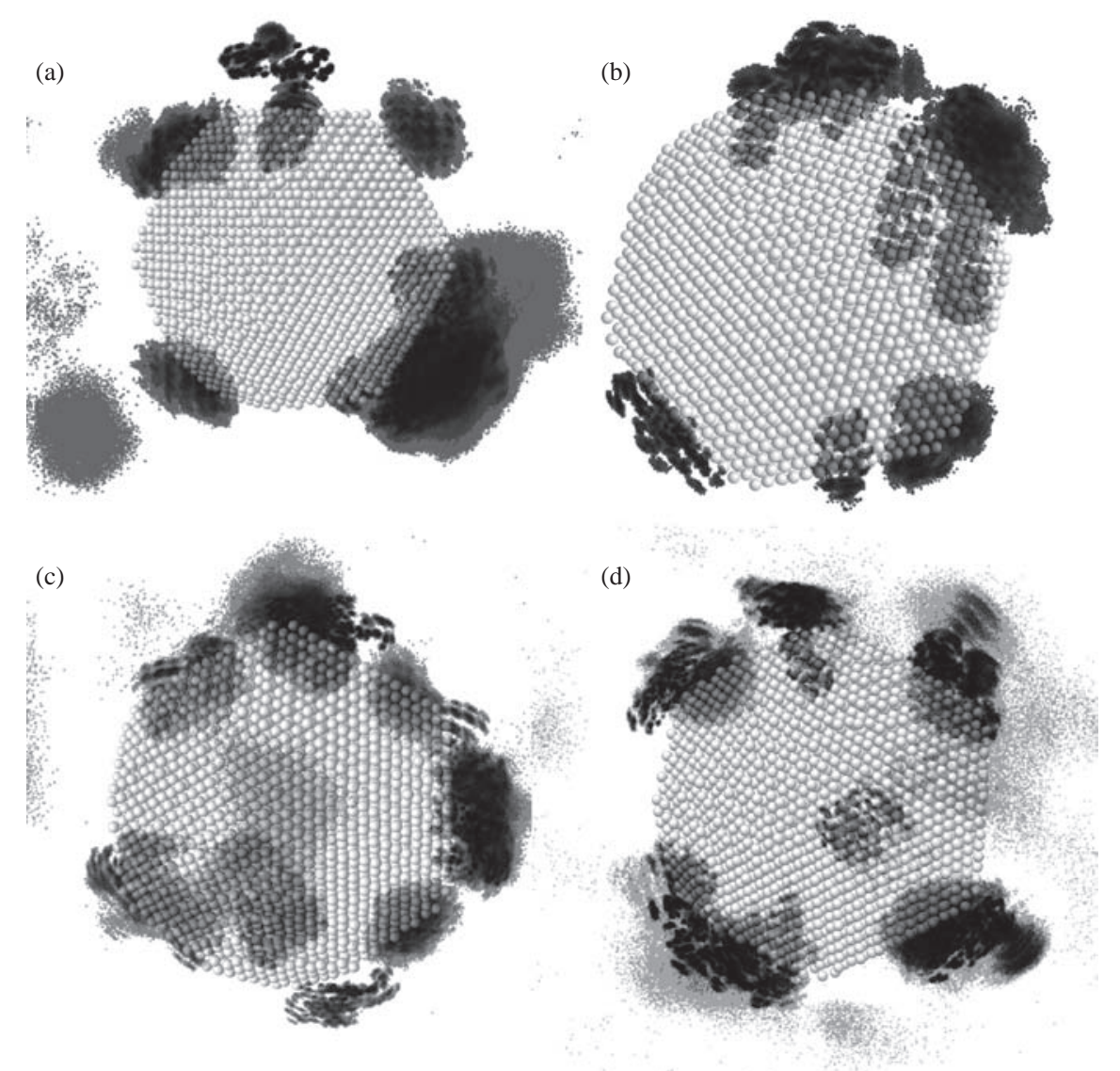

Fig. 3 Density maps of the four active compounds throughout the 30 ns simulation: (a) Isorosmanol, (b) carnosol, (c) rosmanol, and (d) carnosic acid. 


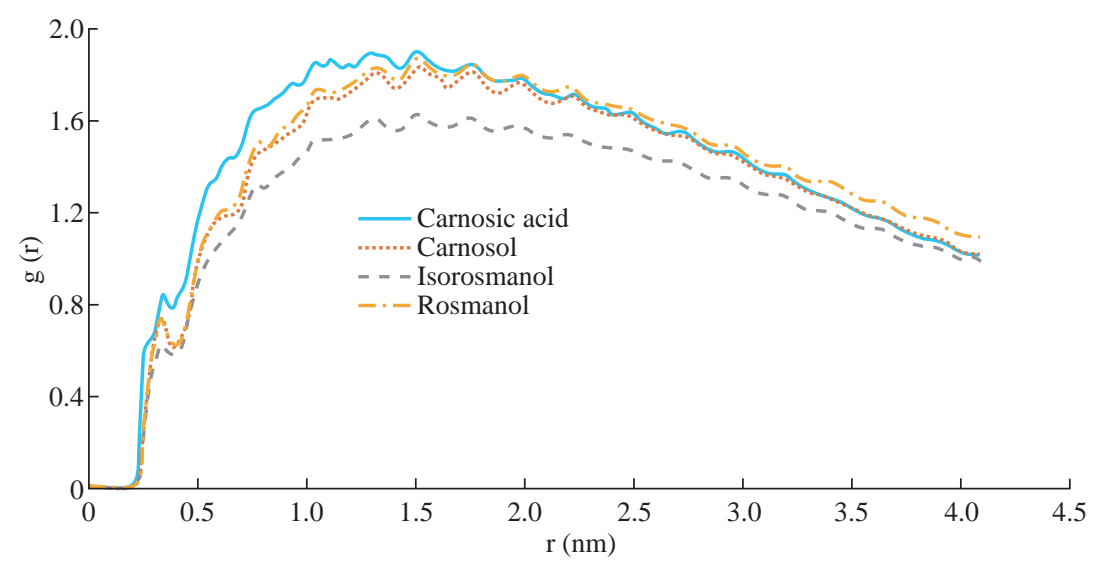

Fig. 4 The radial distribution function of the four active compounds during $30 \mathrm{~ns}$ simulation.

methods have recently been developed to calculate the atomic partial charges of a molecule,however, none are adequately qualified for large molecules or complex structures like NPs. Solving quantum mechanics equations even for a single $\mathrm{Ag}$ atom needs ample computer resources. Considering that an Agconjugated NP contains thousands of atoms, it is almost impossible to accurately calculate its partial charges even by advanced computers [50]. Therefore, we set the atomic partial charges of NP to zero, which provides an approximate model of Ag NP in hand [51, 52].

\section{Experimental synthesis of silver nanoparticles}

Chemical reduction of $\mathrm{Ag}^{+}$to $\mathrm{Ag}-\mathrm{NPs}$ during exposure to $1 \%$ ethanolic extract of Rosmarinus officinalis L. characterized by the color change of solution from yellow to dark brown after addition of $\mathrm{AgNO}_{3}$ is presented in Fig. 5. The color changes are basically caused by a quantum phenomenon that occurs in metallic NPs called excitation of surface plasmon resonance [53, 54].

The exact mechanism of biological reduction of $\mathrm{Ag}^{+}$ to $\mathrm{Ag}$ is not completely understood. The reduction of
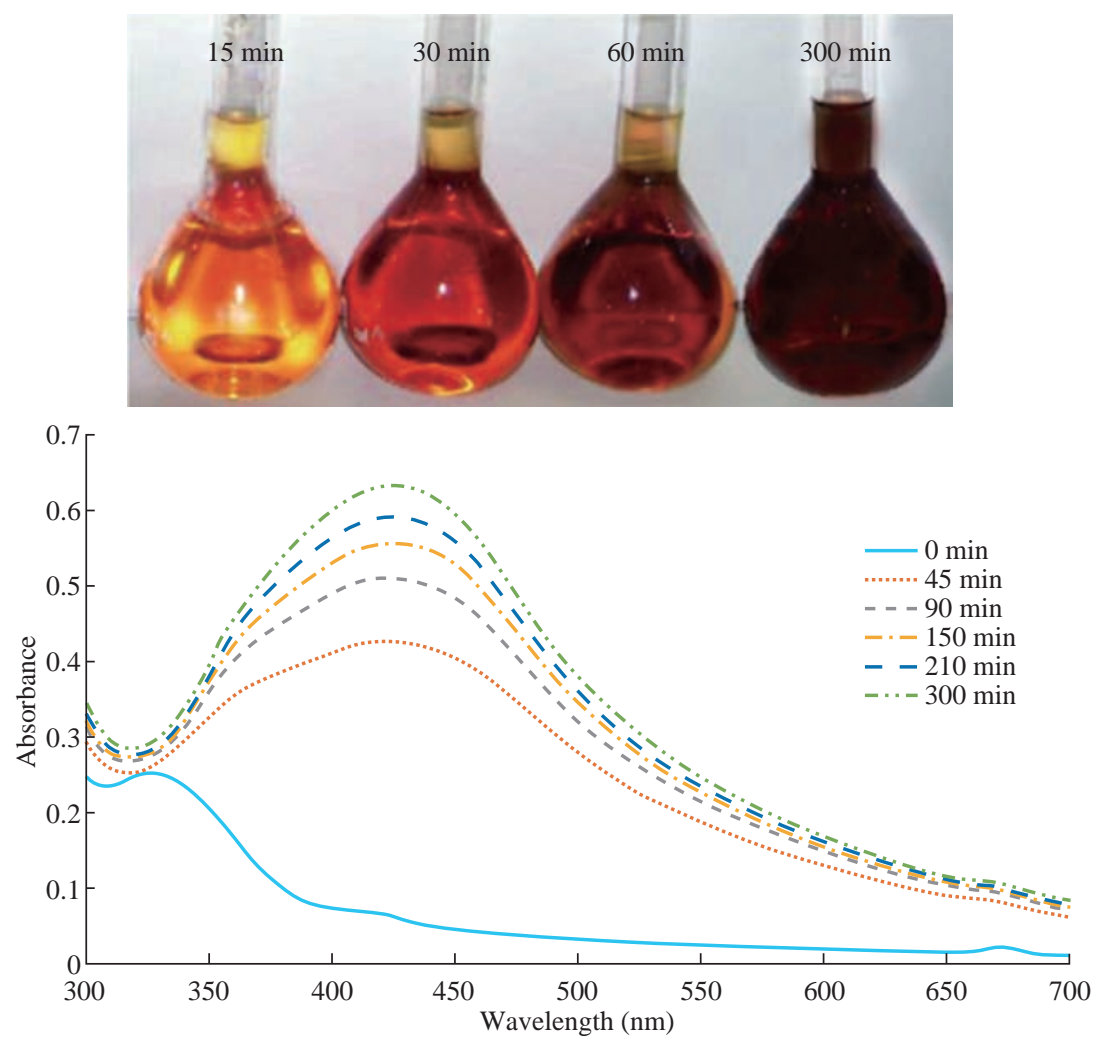

Fig. 5 The ultraviolet-visible spectra of Ag-NPs synthesized by the reduction of silver ions using rosemary leaf extract. The color change of reaction mixtures is depicted on the graph. 
$\mathrm{Ag}^{+}$can happen via reducing agents present in plant extracts such as proteins, secondary metabolites and biologically active compounds, which can also coat the Ag-conjugated NPs and prevent their aggregation. Recently, synthesized stable monodisperse Ag-protein (core-shell) NPs showed an effective antimicrobial potency against two representative bacteria, Staphylococcus aureus and Klebsiella pneumonia. Proteins bind to the NPs either through their free amine groups or cysteine residues $[55,56]$. As it was demonstrated in MD simulation, active compounds and secondary metabolites, can bind to the NPs too. The transmission electron microscopy (TEM) images showed that the size of the synthesized NPs varied between 3 to $30 \mathrm{~nm}$ in diameter, with an average size of 12-15 $\mathrm{nm}$ and a spherical shape (Fig. 6).

\section{Fourier-transform infrared spectroscopy}

Fourier-transform infrared spectroscopy (FTIR) of the biosynthesized Ag-conjugated NPs by rosemary leaf extract suggested that several active compounds present in the extract were attached onto the surface of the synthesized NPs (Fig. 7(a) and Table 1). In the FTIR spectrum, several strong, broad, sharp and weak peaks were observed. The broad and strong peak at $3433 \mathrm{~cm}^{-1}$ represented $\mathrm{O}-\mathrm{H}$ bond stretching in alcohols and phenols which corresponded to the $\mathrm{O}-\mathrm{H}$ and phenol groups in the rosemary's active compounds.
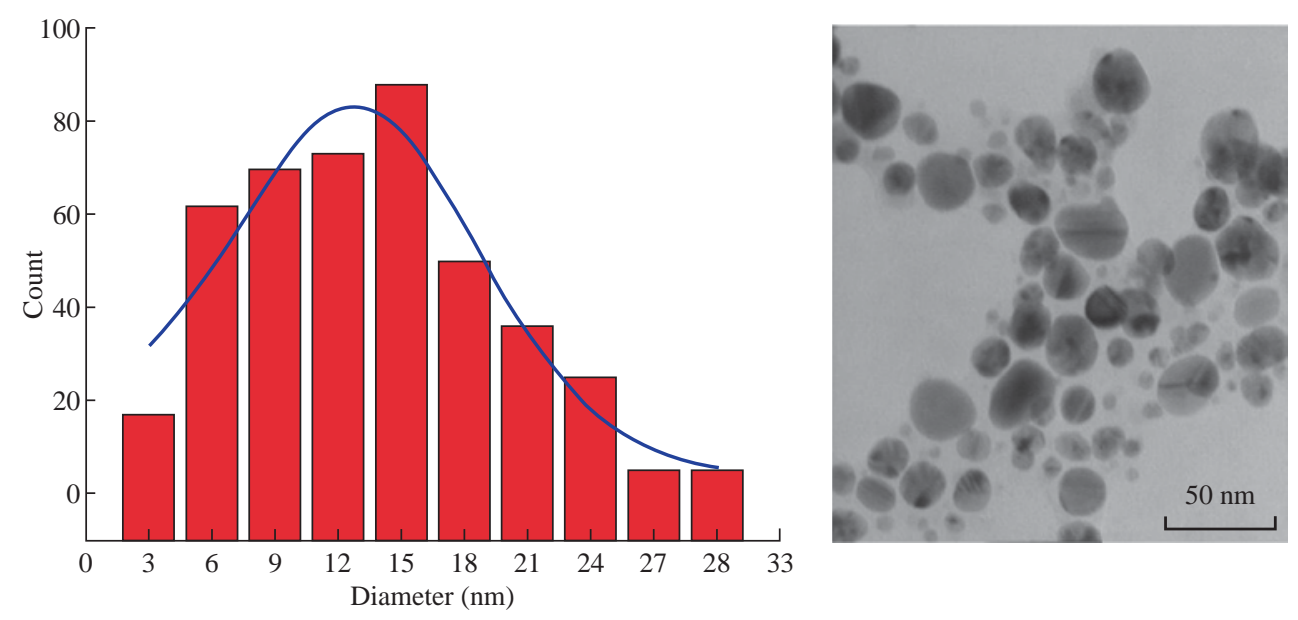

Fig. 6 The transmission electron microscopy image of the synthesized Ag nanoparticles with an average diameter of 12 to $15 \mathrm{~nm}$.
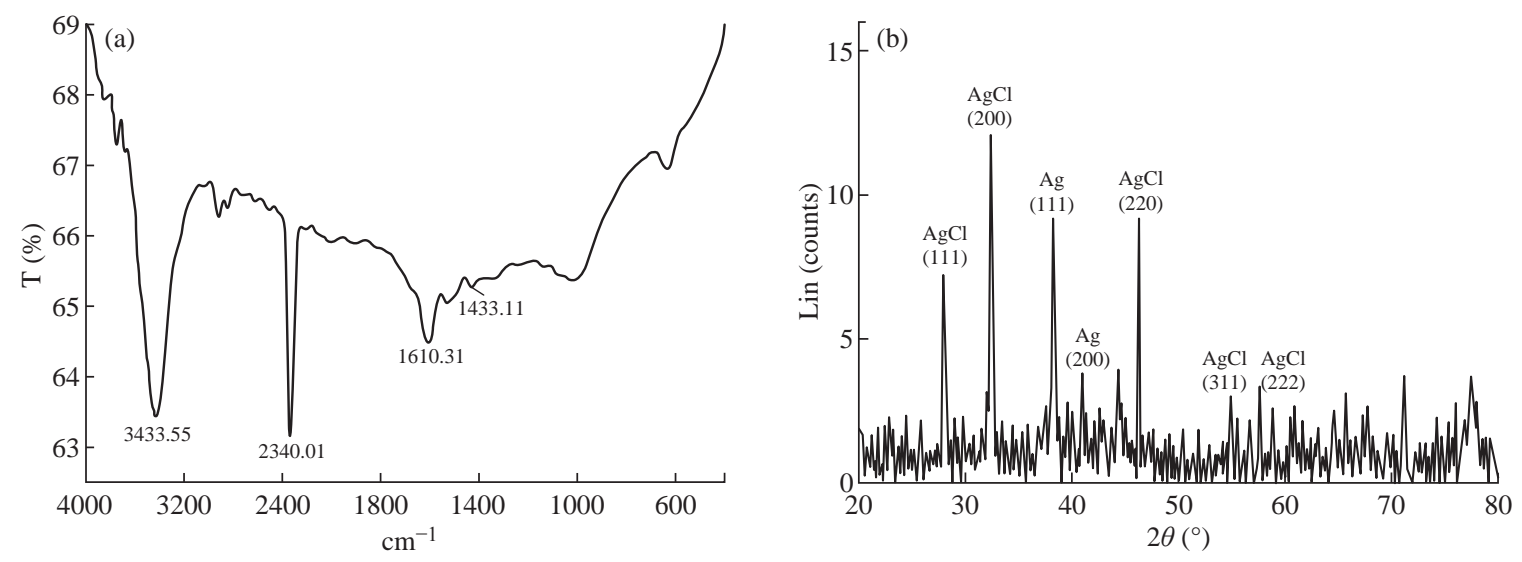

Fig. 7 (a) The Fourier-transform infrared spectrum of synthesized $\mathrm{Ag} / \mathrm{AgCl}-\mathrm{NPs}$. (b) X-ray powder diffraction pattern of $\mathrm{Ag} / \mathrm{AgCl}$ NPs which represented the presence of both $\mathrm{Ag}$ and $\mathrm{AgCl}$ atoms in the solution.

Table 1 The details of the frequencies and the corresponding bonds

\begin{tabular}{cccc}
\hline Absorption $\left(\mathrm{cm}^{-1}\right)$ & Group & Compound class & Appearance \\
\hline 3433 & O-H stretching & alcohol, phenol & strong, broad \\
2340 & $\mathrm{O}=\mathrm{C}=\mathrm{O}$ stretching & carbon dioxide & strong, sharp \\
1610 & $\mathrm{C}=\mathrm{C}$ stretching & cyclic alkene & medium \\
1433 & $\mathrm{O}-\mathrm{H}$ bending & carboxylic acid & medium \\
\hline
\end{tabular}


The $2340 \mathrm{~cm}^{-1}$ strong and sharp peak might probably be related to $\mathrm{CO}_{2}$ contamination. The sharp and weak peak at $1610 \mathrm{~cm}^{-1}$ might be related to the $\mathrm{C}-\mathrm{H}$ bond in the aromatic stretching, associated with the phenolic ring structures or it could be related to aromatic rings which might be indicative of the aromatic amino acids of various proteins, or the aromatic rings of the rosemary's diterpenes. The FTIR spectra also indicated the non-bonded chemical interactions between rosemary leaf extracts and Ag-conjugated NPs.

These observations suggest that several rosemary's metabolites may act as bio-reductants and/or bio-stabilizers. Moreover, the NPs coated with these metabolites may exhibit unique therapeutic properties.

\section{X-ray powder diffraction analysis}

In order to understand the nature and composition of the synthesized Ag-conjugated NPs, X-ray powder diffraction (XRD) analysis was performed, which is indicative of the presence of different atoms in the solution based on the observed peak patterns (Fig. 7(b)). The (111), (200), (220), (222) and (311) peaks represented the $\mathrm{AgCl}$ molecules in the solution and the (111) and (200) peaks represented Ag atoms [57], suggesting that in addition to Ag-NPs, AgCl-NPs were present in the solution (Fig. 7(b)). Similar results were reported when other bio-based NP biosynthesis methods were employed [57, 58].

\section{Conclusions}

Recently, NPs have immensely influenced human lives, especially considering their applications in drug delivery. Data obtained from molecular dynamics simulation of the coating process of Ag-conjugated NP by rosemary's active compounds, revealed that rosmanol, isorosmanol, carnosol, and carnosic acid were able to successfully attach to the surface of the synthesized NPs, probably due to their hydrophobic properties. The in-silico biosynthesis modelling was confirmed by experimental data, and indicated that the synthesized NPs consisted of $\mathrm{Ag} / \mathrm{AgCl}$ with a spherical shape and an average size of 12-15 nm, coated with several bioactive compounds of rosemary leaf extract. Therefore, a new idea is presented for taking advantage of plants' abundancy for biosynthesis of NPs coated with desired active compounds, with promising beneficial applications in future drug discovery approaches.

\section{Acknowledgements}

The authors are very grateful to Stephen Cork (Charles Sturt University, Australia) for providing constructive comments during manuscript preparation. This study was funded by Golestan University.

\section{Conflict of Interests}

The authors declare that no competing interest exists.

\section{References}

[1] W.H. De Jong, P.J. Borm, Drug delivery and nanoparticles:applications and hazards. Int $J$ Nanomedicine, 2008, 3(2): 133-149.

[2] J.K. Patra, G. Das, L.F. Fraceto, et al., Nano based drug delivery systems: recent developments and future prospects. Journal of Nanobiotechnology, 2018, 16 (1): 71.

[3] J. Wang, X. Hu, and D. Xiang, Nanoparticle drug delivery systems: an excellent carrier for tumor peptide vaccines. Drug Deliv, 2018, 25(1): 1319-1327.

[4] S. ravani, H. Korbekandi, S.V. Mirmohammadi, et al., Synthesis of silver nanoparticles: chemical, physical and biological methods. Res Pharm Sci, 2014, 9(6): 385-406

[5] M.A. Sabri, A. Umer, G.H. Awan, et al., Selection of Suitable Biological Method for the Synthesis of Silver Nanoparticles. Nanomaterials and Nanotechnology, 2016, 6: 29.

[6] R.A. Hamouda, M.H. Hussein, R.A. Abo-elmagd, et al., Synthesis and biological characterization of silver nanoparticles derived from the cyanobacterium Oscillatoria limnetica. Scientific Reports, 2019, 9(1): 13071.

[7] A. Khandelwal, R. Joshi, P. Mukherjee, et al., Use of bio-based nanoparticles in agriculture. Nanotechnology for agriculture: Advances for sustainable agriculture. Springer Singapore, 2019: 89-100.

[8] V.V. Makarov, A.J. Love, O.V. Sinitsyna, et al., "Green" nanotechnologies: synthesis of metal nanoparticles using plants. Acta Naturae, 2014, 6(1): 35-44.

[9] I.M. Chung, I. Park, K. Seung-Hyun, et al., PlantMediated Synthesis of Silver Nanoparticles: Their Characteristic Properties and Therapeutic Applications. Nanoscale Res Lett, 2016, 11(1): 40.

[10] A.K. Mittal, Y. Chisti, and U.C. Banerjee, Synthesis of metallic nanoparticles using plant extracts. Biotechnology Advances, 2013, 31(2): 346-356.

[11] E. Kohan Baghkheirati, M.B. Bagherieh-Najjar, H. Khandan Fadafan, et al., Synthesis and antibacterial activity of stable bio-conjugated nanoparticles mediated by walnut (Juglans regia) green husk extract. Journal of Experimental Nanoscience, 2016, 11(7): 512-517.

[12] K Jemal, B. Sandeep, and S.P. Pola, Synthesis, characterization, and evaluation of the antibacterial activity of Allophylus serratus leaf and leaf derived Callus extracts mediated silver nanoparticles. Journal of Nanomaterials, 2017, 2017: 1-11.

[13] J.J. Lee, Y.R. Jin, J.H. Lee, et al., Antiplatelet activity of carnosic acid, a phenolic diterpene from Rosmarinus officinalis. Planta Med, 2007, 73(2): 121-127.

[14] L. D’Evoli, L. Huikko, A.M. Lampi, et al., Influence 
of rosemary (Rosmarinus officinalis, L.) on plant sterol oxidation in extra virgin olive oil. Mol Nutr Food Res, 2006, 50(9): 818-823.

[15] S.J. Posadas, V. Caz, C. Largo, et al., Protective effect of supercritical fluid rosemary extract, Rosmarinus officinalis, on antioxidants of major organs of aged rats. Experimental Gerontology, 2009, 44(6): 383-389.

[16] C.H. Peng, J.D. Su, C.C. Chyau, et al., Supercritical fluid extracts of rosemary leaves exhibit potent antiinflammation and anti-tumor effects. Biosci Biotechnol Biochem, 2007, 71(9): 2223-2232.

[17] I. Takaki, L.E. Bersani-Amado, A. Vendruscolo, et al., Anti-inflammatory and antinociceptive effects of Rosmarinus officinalis L. essential oil in experimental animal models. J Med Food, 2008, 11(4): 741-746.

[18] T. Bakırel, U. Bakırel, O.Ü. Keleş, et al., In vivo assessment of antidiabetic and antioxidant activities of rosemary (Rosmarinus officinalis) in alloxan-diabetic rabbits. Journal of Ethnopharmacology, 2008, 116(1): 6473.

[19] H.C. Huang, C.Y. Huang, S.Y. Lin-Shiau, et al., Ursolic acid inhibits IL-1beta or TNF-alpha-induced C6 glioma invasion through suppressing the association ZIP/p62 with PKC-zeta and downregulating the MMP-9 expression. Mol Carcinog, 2009, 48(6): 517-531.

[20] M.A. Del Nobile, A. Lucera, C. Costa, et al., Food applications of natural antimicrobial compounds. Frontiers in Microbiology, 2012, 3(287).

[21] M.M. Özcan, J.C. Chalchat, Chemical composition and antifungal activity of rosemary (Rosmarinus officinalis L.) oil from Turkey. International Journal of Food Sciences and Nutrition, 2008, 59(7-8): 691-698.

[22] A. Kovi, I. Milanovi, N. Pavlović, et al., Antioxidant activity of rosemary (Rosmarinus officinalis L.) essential oil and its hepatoprotective potential. $B M C$ Complementary and Alternative Medicine, 2014, 14: 225.

[23] S. Habtemariam, The therapeutic potential of rosemary (Rosmarinus officinalis) diterpenes for Alzheimer's disease. Evid Based Complement Alternat Med, 2016, 2016: 2680409.

[24] S.M. Petiwala, J.J. Johnson, Diterpenes from rosemary (Rosmarinus officinalis): Defining their potential for anticancer activity. Cancer Letters, 2015, 367(2): 93-102.

[25] Y. Shan, E.T. Kim, M.P. Eastwood, et al., How Does a Drug Molecule Find Its Target Binding Site? Journal of the American Chemical Society, 2011, 133(24): 91819183.

[26] A. Kyrychenko, O.M. Korsun, I.I. Gubin, et al., Atomistic simulations of coating of silver nanoparticles with poly(vinylpyrrolidone) oligomers: Effect of oligomer chain length. The Journal of Physical Chemistry C, 2015, 119(14): 7888-7899.

[27] S. Pronk, S. Páll, R. Schulz, et al., GROMACS 4.5: A high-throughput and highly parallel open source molecular simulation toolkit. Bioinformatics, 2013, 29(7): 845-854.

[28] C. Oostenbrink, A. Villa, A.E. Mark, et al., A biomolecular force field based on the free enthalpy of hydration and solvation: The GROMOS force-field parameter sets 53A5 and 53A6. Journal of Computational Chemistry, 2004, 25(13): 1656-1676.

[29] W.L. Jorgensen, J. Chandrasekhar, J.D. Madura, et al., Comparison of simple potential functions for simulating liquid water. The Journal of Chemical Physics, 1983, 79(2): 926-935.

[30] S.A. Adcock, J.A. McCammon, Molecular dynamics: Survey of methods for simulating the activity of proteins. Chem Rev, 2006, 106(5): 1589-1615.

[31] B. Hess, H. Bekker, H.J.C. Berendsen, et al., LINCS: A linear constraint solver for molecular simulations. Journal of Computational Chemistry, 1997, 18(12): 1463-1472.

[32] U. Essmann, L. Perera, M.L. Berkowitz, et al., A smooth particle mesh Ewald method. The Journal of Chemical Physics, 1995, 103(19): 8577-8593.

[33] S. Miyamoto, P.A. Kollman, Settle: An analytical version of the SHAKE and RATTLE algorithm for rigid water models. Journal of Computational Chemistry, 1992, 13(8):952-962.

[34] W. Humphrey, A. Dalke, and K. Schulten, VMD: Visual molecular dynamics. Journal of Molecular Graphics, 1996, 14(1): 33-38.

[35] E.F. Pettersen, T.D. Goddard, C.C. Huang, et al., UCSF Chimera - A visualization system for exploratory research and analysis. J Comput Chem, 2004, 25(13): 1605-1612.

[36] A.K. Malde, L. Zuo, M. Breeze, et al., An automated force field topology builder (ATB) and repository: Version 1.0. J Chem Theory Comput, 2011, 7(12): 4026-4037.

[37] H. Heinz, R.A. Vaia, B.L. Farmer, et al., Accurate simulation of surfaces and interfaces of face-centered cubic metals using 12-6 and 9-6 Lennard-Jones potentials. The Journal of Physical Chemistry C, 2008, 112(44): 17281-17290.

[38] K. Duanmu, D.G. Truhlar, Partial ionic character beyond the Pauling paradigm: Metal nanoparticles. The Journal of Physical Chemistry C, 2014, 118(48): 28069-28074.

[39] M. Hadi, M. Bagherieh-Najjar, E. Kohan, et al., AgConjugated Nanoparticle Biosynthesis Mediated by Rosemary Leaf Extracts Correlates with Plant Antioxidant Activity and Protein Content. 2018.

[40] T. Nezamdoost, M.B. Bagherieh-Najjar, and M. Aghdasi, Biogenic synthesis of stable bioactive silver chloride nanoparticles using Onosma dichroantha Boiss. root extract. Materials Letters, 2014, 137: 225-228.

[41] J.L. Rosas-Trigueros, J. Correa-Basurto, C.G. BenitezCardoza, et al., Insights into the structural stability of Bax from molecular dynamics simulations at high temperatures. Protein Sci, 2011, 20(12): 2035-2046.

[42] L. Esposito, A. Paladino, C. Pedone, et al., Insights into structure, stability, and toxicity of monomeric and aggregated polyglutamine models from molecular dynamics simulations. Biophysical Journal, 2008, 94(10): 4031-4040.

[43] D. Zhang, R. Lazim, Application of conventional molecular dynamics simulation in evaluating the stability of apomyoglobin in urea solution. Sci Rep, 2017, 7: 44651.

[44] M. Sharma, C.R. Anirudh, Mechanism of mRNA-STAR domain interaction: Molecular dynamics simulations of Mammalian Quaking STAR protein. Scientific Reports, 2017, 7 (1): 12567.

[45] M. Kurciński, M. Jarończyk, F.J.P. Lipiński, et al., Structural insights into $\sigma 1$ receptor interactions with opioid ligands by molecular dynamics simulations. Molecules, 2018, 23(2).

[46] H. Zhang, T. Song, Y. Yang, et al., Exploring the interaction mechanism between cyclopeptide DC3 and androgen receptor using molecular dynamics simulations and free energy calculations. Front Chem, 2018, 6: 119.

[47] D.E. Elmore, Molecular dynamics simulation of a phosphatidylglycerol membrane. FEBS Letters, 2006, 580(1): 144-148.

[48] L.-W. Wang, Divide-and-conquer quantum mechanical material simulations with exascale supercomputers. National Science Review, 2014, 1(4): 604-617.

[49] Y. Zhao, D. Liu, H. Tang, et al., A MD simulation and analysis for aggregation behaviors of nanoscale zerovalent iron particles in water via MS. The Scientific World Journal, 2014, 2014: 768780.

[50] D.W. Borhani, D.E. Shaw, The future of molecular dynamics simulations in drug discovery. J Comput Aided 
Mol Des, 2012, 26(1): 15-26.

[51] S. Riniker, Fixed-charge atomistic force fields for molecular dynamics simulations in the condensed phase: An overview. Journal of Chemical Information and Modeling, 2018, 58(3): 565-578.

[52] L. Ye, J. Liu, C. Gong, et al., Two different roles of metallic $\mathrm{Ag}$ on $\mathrm{Ag} / \mathrm{AgX} / \mathrm{BiOX}(\mathrm{X}=\mathrm{Cl}, \mathrm{Br})$ visible light photocatalysts: Surface plasmon resonance and Z-scheme bridge. ACS Catalysis, 2012, 2(8): 1677-1683.

[53] P. Mulvaney, Surface plasmon spectroscopy of nanosized metal particles. Langmuir, 1996, 12(3): 788-800.

[54] S. Li, Y. Shen, A. Xie, et al., Green synthesis of silver nanoparticles using Capsicum annuum L. extract. Green Chemistry - GREEN CHEM, 2007, 9.

[55] A. Gole, C. Dash, V. Ramakrishnan, et al., Pepsin-gold colloid conjugates: Preparation, characterization, and enzymatic activity. Langmuir, 2001, 17(5): 1674-1679.

[56] K.N. Thakkar, S.S. Mhatre, and R.Y. Parikh, Biological synthesis of metallic nanoparticles. Nanomedicine, 2010,
6(2): 257-262.

[57] C. Han, L. Ge, C. Chen, et al., Site-selected synthesis of novel $\mathrm{Ag} @ \mathrm{AgCl}$ nanoframes with efficient visible light induced photocatalytic activity. Journal of Materials Chemistry A, 2014, 2(31): 12594-12600.

[58] M.S. Al Aboody, Silver/silver chloride (Ag/AgCl) nanoparticles synthesized from Azadirachta indica lalex and its antibiofilm activity against fluconazole resistant Candida tropicalis. Artificial Cells, Nanomedicine, and Biotechnology, 2019, 47(1): 2107-2113.

Copyright $\subseteq$ Farzin Sohraby, Mojtaba Hadi Soltanabad, Milad Bagheri, Masood Bezi Javan, Mostafa Javaheri Moghadam, Eisa Kohan Baghkheirati, and Mohammad Bagher Bagherieh Najjar. This is an open-access article distributed under the terms of the Creative Commons Attribution License, which permits unrestricted use, distribution, and reproduction in any medium, provided the original author and source are credited. 